\title{
On the well-posedness of the semi-relativistic Schrödinger-Poisson system
}

\author{
Walid Abou Salem, Thomas Chen, and Vitali Vougalter
}

Communicated by $Y$. Charles Li, received December 15, 2011.

\begin{abstract}
We show global existence and uniqueness of strong solutions for the Schrödinger-Poisson system in the repulsive Coulomb case with relativistic kinetic energy.
\end{abstract}

\section{Contents}

1. Introduction 121

2. Proof of global well-posedness 123

$\begin{array}{ll}\text { References } & 132\end{array}$

\section{Introduction}

In this article, we study the global well-posedness of the semi-relativistic SchrödingerPoisson system on a finite domain. This system is relevant to the description of many-body semi-relativistic quantum particles in the mean-field limit (for instance, in heated plasma), when the particles move with extremely high velocities. Consider semi-relativistic quantum particles confined in domain $\Omega \subset \mathbb{R}^{3}$ which is an open, finite volume set with a $C^{2}$ boundary. The particles interact by the electrostatic field they collectively generate. In the mean-field limit, the density matrix that describes the mixed state of the system satisfies the Hartree-von Neumann equation

$$
\left\{\begin{array}{l}
i \partial_{t} \rho(t)=\left[H_{V}, \rho(t)\right], \quad x \in \Omega, \quad t \geq 0 \\
-\Delta V=n(t, x), \quad n(t, x)=\rho(t, x, x), \quad \rho(0)=\rho_{0}
\end{array}\right.
$$

1991 Mathematics Subject Classification. 82D10, 82C10.

Key words and phrases. Schrödinger-Poisson system, functional spaces, density matrices, global existence and uniqueness. 
satisfying Dirichlet boundary conditions, $\rho(t, x, y)=0$ if $x$ or $y \in \partial \Omega$, for $t \geq 0$. The Hamiltonian is given by

$$
H_{V}:=T_{m}+V(t, x)
$$

where the relativistic kinetic energy operator $T_{m}:=\sqrt{-\Delta+m^{2}}-m$ is defined via the spectral calculus. Here, $\Delta$ denotes the Dirichlet Laplacian on $L^{2}(\Omega)$, and $m>0$ is the particle mass; see $[\mathbf{3}, \mathbf{2}]$ for a derivation of this system of equations in the non-relativistic case. Since $\rho(t)$ is a positive, self-adjoint trace-class operator acting on $L^{2}(\Omega)$, its kernel can, for every $t \in \mathbb{R}_{+}$, be decomposed with respect to an orthonormal basis of $L^{2}(\Omega)$. The kernel of the initial data $\rho_{0}$ can be represented in the form

$$
\rho_{0}(x, y)=\sum_{k \in \mathbb{N}} \lambda_{k} \psi_{k}(x) \overline{\psi_{k}(y)}
$$

where $\left\{\psi_{k}\right\}_{k \in \mathbb{N}}$ denotes an orthonormal basis of $L^{2}(\Omega)$, with $\left.\psi_{k}\right|_{\partial \Omega}=0$ for all $k \in \mathbb{N}$, and coefficients

$$
\underline{\lambda}:=\left\{\lambda_{k}\right\}_{k \in \mathbb{N}} \in \ell^{1}, \quad \lambda_{k} \geq 0, \quad \sum_{k} \lambda_{k}=1 .
$$

As shown below, there exists a one-parameter family of complete orthonormal bases of $L^{2}(\Omega),\left\{\psi_{k}(t)\right\}_{k \in \mathbb{N}}$, with $\left.\psi_{k}(t)\right|_{\partial \Omega}=0$ for all $k \in \mathbb{N}$, and for $t \in \mathbb{R}_{+}$, such that the kernel of the solution $\rho(t)$ to (1.1) can be represented as

$$
\rho(t, x, y)=\sum_{k \in \mathbb{N}} \lambda_{k} \psi_{k}(t, x) \overline{\psi_{k}(t, y)}
$$

Notably, the coefficients $\underline{\lambda}$ are independent of $t$, and thus the same as those in $\rho_{0}$. Substituting (1.5) in (1.1), the one-parameter family of orthonormal vectors $\left\{\psi_{k}(t)\right\}_{k \in \mathbb{N}}$ is seen to satisfy the semi-relativistic Schrödinger-Poisson system

$$
\begin{gathered}
i \frac{\partial \psi_{k}}{\partial t}=T_{m} \psi_{k}+V \psi_{k}, \quad k \in \mathbb{N} \\
-\Delta V[\Psi]=n[\Psi], \quad \Psi:=\left\{\psi_{k}\right\}_{k=1}^{\infty}, \\
n[\Psi(x, t)]=\sum_{k=1}^{\infty} \lambda_{k}\left|\psi_{k}\right|^{2},
\end{gathered}
$$

with initial data $\left\{\psi_{k}(0)\right\}_{k=1}^{\infty}$. The potential function $V[\Psi]$ solves the Poisson equation (1.7). On both $V[\Psi]$ and $\psi_{k}(t)$, for all $k \in \mathbb{N}$, we impose Dirichlet boundary conditions

$$
\psi_{k}(t, x), \quad V(x, t)=0, t \geq 0, \forall x \in \partial \Omega .
$$

As we show in Lemma 6, below, solutions of (1.6)-(1.8) preserve the orthonormality of $\left\{\psi_{k}(t)\right\}_{k \in \mathbb{N}}$.

The state space for the Schrödinger-Poisson system is given by

$$
\begin{aligned}
\mathcal{L}:= & \left\{(\Psi, \underline{\lambda}) \mid \Psi=\left\{\psi_{k}\right\}_{k=1}^{\infty} \subset H_{0}^{\frac{1}{2}}(\Omega) \cap H^{1}(\Omega)\right. \text { is a complete } \\
& \text { orthonormal system in } L^{2}(\Omega), \\
& \left.\underline{\lambda}=\left\{\lambda_{k}\right\}_{k=1}^{\infty} \in \ell^{1}, \quad \lambda_{k} \geq 0, k \in \mathbb{N}, \quad \sum_{k=1}^{\infty} \lambda_{k} \int_{\Omega}\left|\nabla \psi_{k}\right|^{2} d x<\infty\right\} .
\end{aligned}
$$


For fixed $\underline{\lambda} \in \ell^{1}, \quad \lambda_{k}>0$, and for sequences of square integrable functions $\Phi:=$ $\left\{\phi_{k}\right\}_{k=1}^{\infty}$ and $\Psi:=\left\{\psi_{k}\right\}_{k=1}^{\infty}$, we define the inner product

$$
(\Phi, \Psi)_{\mathcal{L}_{\underline{\underline{\lambda}}}^{2}(\Omega)}:=\sum_{k=1}^{\infty} \lambda_{k}\left(\phi_{k}, \psi_{k}\right)_{L^{2}(\Omega)},
$$

which induces the norm

$$
\|\Phi\|_{\mathcal{L}_{\underline{\underline{\lambda}}}^{2}(\Omega)}:=\left(\sum_{k=1}^{\infty} \lambda_{k}\left\|\phi_{k}\right\|_{L^{2}(\Omega)}^{2}\right)^{\frac{1}{2}},
$$

and we introduce the corresponding Hilbert space

$$
\mathcal{L}_{\underline{\underline{\lambda}}}^{2}(\Omega):=\left\{\Phi=\left\{\phi_{k}\right\}_{k=1}^{\infty} \mid \phi_{k} \in L^{2}(\Omega), \forall k \in \mathbb{N},\|\Phi\|_{\mathcal{L}_{\underline{\lambda}}^{2}(\Omega)}<\infty\right\} .
$$

Our main result is as follows.

Theorem 1. For every initial state $(\Psi(x, 0), \underline{\lambda}) \in \mathcal{L}$, there is a unique mild solution $\Psi(x, t), t \in[0, \infty)$, of (1.6)-(1.8) with $(\Psi(x, t), \underline{\lambda}) \in \mathcal{L}$, which is also a unique strong global solution in $\mathcal{L}_{\underline{\underline{\lambda}}}^{2}(\Omega)$.

Establishing the global well-posedness of the Schrödinger-Poisson system plays a crucial role in proving the existence and nonlinear stability of stationary states, i.e. the nonlinear bound states of the Schrödinger-Poisson system, which was done in the nonrelativistic case in $[\mathbf{4}, \mathbf{6}]$. The problem in one dimension was treated in [8]. The semiclassical limit of the Schrödinger-Poisson system with the relativistic kinetic energy was studied in the recent article [1]. Global well-posedness for a single semi-relativistic Hartree equation in $\mathbb{R}^{3}$ was established in [5]. In the present work, we deal with the infinite system of equations in a finite volume set with Dirichlet boundary conditions, and, as distinct from [5], we do not use the regularization of the Poisson equation. Moreover, both the results of [5] and Theorem 1 above do not rely on Strichartz type estimates.

\section{Proof of global well-posedness}

We make a fixed choice of $\underline{\lambda}=\left\{\lambda_{k}\right\}_{k=1}^{\infty} \in \ell^{1}$, with $\lambda_{k}>0$ and $\sum \lambda_{k}=$ 1 , denoting the sequence of coefficients determined by the initial data $\rho_{0}$ of the Hartree-von Neumann equation (1.1) via (1.5), for $t=0$. We note that we require all $\lambda_{k}>0$ to be positive for the subsequent analysis. This does not lead to any loss of generality since by density arguments, any $\rho_{0}$ (and likewise $\rho(t)$ ) can be approximated arbitrarily well by an expansion of the form (1.3), respectively (1.5), with $\lambda_{k}>0$.

We introduce inner products $(\cdot, \cdot)_{\mathcal{H}_{\underline{\lambda}}^{1 / 2}(\Omega)}$ and $(\cdot, \cdot)_{\mathcal{H}_{\underline{\lambda}}^{1}(\Omega)}$ which induce the generalized inhomogenous Sobolev norms

$$
\|\Phi\|_{\mathcal{H}_{\underline{\lambda}}^{1 / 2}(\Omega)}:=\left(\sum_{k=1}^{\infty} \lambda_{k}\left\|\phi_{k}\right\|_{H^{\frac{1}{2}}(\Omega)}^{2}\right)^{\frac{1}{2}} \text { and }\|\Phi\|_{\mathcal{H}_{\underline{\lambda}}^{1}(\Omega)}:=\left(\sum_{k=1}^{\infty} \lambda_{k}\left\|\phi_{k}\right\|_{H^{1}(\Omega)}^{2}\right)^{\frac{1}{2}}
$$

and define the corresponding Hilbert spaces

$$
\mathcal{H}_{\underline{\lambda}}^{1 / 2}(\Omega):=\left\{\Phi=\left\{\phi_{k}\right\}_{k=1}^{\infty} \mid \phi_{k} \in H_{0}^{\frac{1}{2}}(\Omega), \forall k \in \mathbb{N},\|\Phi\|_{\mathcal{H}_{\underline{\lambda}}^{1 / 2}(\Omega)}<\infty\right\}
$$


and

$$
\mathcal{H}_{\underline{\underline{\lambda}}}^{1}(\Omega):=\left\{\Phi=\left\{\phi_{k}\right\}_{k=1}^{\infty} \mid \phi_{k} \in H_{0}^{\frac{1}{2}}(\Omega) \cap H^{1}(\Omega), \forall k \in \mathbb{N},\|\Phi\|_{\mathcal{H}_{\underline{\lambda}}^{1}(\Omega)}<\infty\right\}
$$

respectively. We also introduce the generalized homogenous Sobolev norms

$$
\|\Phi\|_{\dot{\mathcal{H}}_{\underline{\underline{\lambda}}}^{1 / 2}(\Omega)}:=\left(\sum_{k=1}^{\infty} \lambda_{k}\left\||p|^{\frac{1}{2}} \phi_{k}\right\|_{L^{2}(\Omega)}^{2}\right)^{\frac{1}{2}} \text { and }\|\Phi\|_{\dot{\mathcal{H}}_{\underline{\underline{\lambda}}}^{1}(\Omega)}:=\left(\sum_{k=1}^{\infty} \lambda_{k}\left\|\nabla \phi_{k}\right\|_{L^{2}(\Omega)}^{2}\right)^{\frac{1}{2}}
$$

Here, $|p|$ stands for the operator $\sqrt{-\Delta}$, and has the meaning of the relativistic kinetic energy of a particle with zero mass. We note the following equivalence of norms.

LEMMA 2. For $\Phi \in \mathcal{H}_{\underline{\lambda}}^{1 / 2}(\Omega)$, the norms $\|\Phi\|_{\mathcal{H}_{\underline{\underline{\lambda}}}^{1 / 2}(\Omega)}$ and $\|\Phi\|_{\dot{\mathcal{H}}_{\underline{\underline{\lambda}}}^{1 / 2}(\Omega)}$ are equivalent. If $\Phi \in \mathcal{H}_{\underline{\underline{\lambda}}}^{1}(\Omega)$, then $\|\Phi\|_{\mathcal{H}_{\underline{\underline{\lambda}}}^{1}(\Omega)}$ is equivalent to $\|\Phi\|_{\mathcal{H}_{\underline{\underline{\lambda}}}^{1}(\Omega)}$.

Proof. Clearly

$$
\begin{aligned}
& \|\Phi\|_{\mathcal{H}_{\underline{\lambda}}^{1 / 2}(\Omega)} \leq\left(\sum_{k=1}^{\infty} \lambda_{k}\left\{\left\|\phi_{k}\right\|_{L^{2}(\Omega)}^{2}+\left\||p|^{\frac{1}{2}} \phi_{k}\right\|_{L^{2}(\Omega)}^{2}\right\}\right)^{\frac{1}{2}} \\
& =\left(\sum_{k=1}^{\infty} \lambda_{k}\left\|\phi_{k}\right\|_{H^{\frac{1}{2}(\Omega)}}^{2}\right)^{\frac{1}{2}}=\|\Phi\|_{\mathcal{H}_{\underline{\lambda}}^{1 / 2}(\Omega)} .
\end{aligned}
$$

We will make use of the Poincaré inequality

$$
\int_{\Omega}\left|\nabla \phi_{k}\right|^{2} d x \geq c_{p} \int_{\Omega}\left|\phi_{k}\right|^{2} d x
$$

with the constant $c_{p}>0$ dependent upon the domain $\Omega$ with Dirichlet boundary conditions. Thus

which enables us to estimate

$$
\left\||p|^{\frac{1}{2}} \phi_{k}\right\|_{L^{2}(\Omega)}^{2} \geq \sqrt{c_{p}}\left\|\phi_{k}\right\|_{L^{2}(\Omega)}^{2},
$$

$$
\begin{gathered}
\|\Phi\|_{\mathcal{H}_{\underline{\lambda}}^{1 / 2}(\Omega)}=\left(\sum_{k=1}^{\infty} \lambda_{k}\left\{\left\|\phi_{k}\right\|_{L^{2}(\Omega)}^{2}+\left\||p|^{\frac{1}{2}} \phi_{k}\right\|_{L^{2}(\Omega)}^{2}\right\}\right)^{\frac{1}{2}} \leq \\
\leq \sqrt{1+\frac{1}{\sqrt{c_{p}}}}\left(\sum_{k=1}^{\infty} \lambda_{k}\left\||p|^{\frac{1}{2}} \phi_{k}\right\|_{L^{2}(\Omega)}^{2}\right)^{\frac{1}{2}}=C\|\Phi\|_{\dot{\mathcal{H}}_{\underline{\lambda}}^{1 / 2}(\Omega)} .
\end{gathered}
$$

Let us compare the remaining two norms. Clearly,

$$
\|\Phi\|_{\dot{\mathcal{H}}_{\underline{\underline{\lambda}}}^{1}(\Omega)} \leq\left(\sum_{k=1}^{\infty} \lambda_{k}\left\|\phi_{k}\right\|_{H^{1}(\Omega)}^{2}\right)^{\frac{1}{2}}=\|\Phi\|_{\mathcal{H}_{\underline{\lambda}}^{1}(\Omega)} .
$$

On the other hand, by means of the Poincaré inequality (2.1),

$$
\begin{gathered}
\|\Phi\|_{\mathcal{H}_{\underline{\lambda}}^{1}(\Omega)}=\left(\sum_{k=1}^{\infty} \lambda_{k}\left\{\left\|\phi_{k}\right\|_{L^{2}(\Omega)}^{2}+\left\|\nabla \phi_{k}\right\|_{L^{2}(\Omega)}^{2}\right\}\right)^{\frac{1}{2}} \leq \\
\leq \sqrt{1+\frac{1}{c_{p}}}\left(\sum_{k=1}^{\infty} \lambda_{k}\left\|\nabla \phi_{k}\right\|_{L^{2}(\Omega)}^{2}\right)^{\frac{1}{2}}=\|\Phi\|_{\dot{\mathcal{H}}_{\underline{\lambda}}^{1}(\Omega)} .
\end{gathered}
$$


Let $\Psi=\left\{\psi_{m}\right\}_{m=1}^{\infty}$ be a wave function and the relativistic kinetic energy operator acts on it $T_{m} \Psi=\left(\sqrt{-\Delta+m^{2}}-m\right) \psi$ componentwise. We have the following two lemmas.

Lemma 3. The domain of the kinetic energy operator is given by $D\left(T_{m}\right)=$ $\mathcal{H}_{\underline{\lambda}}^{1}(\Omega) \subseteq \mathcal{L}_{\underline{\lambda}}^{2}(\Omega)$.

Proof. Let $\Psi \in \mathcal{H}_{\underline{\lambda}}^{1}(\Omega)$. Then

$$
\sum_{m=1}^{\infty} \lambda_{m}\left\|\psi_{m}\right\|_{H^{1}(\Omega)}^{2}=\sum_{m=1}^{\infty} \lambda_{m}\left\{\left\|\psi_{m}\right\|_{L^{2}(\Omega)}^{2}+\left\|\nabla \psi_{m}\right\|_{L^{2}(\Omega)}^{2}\right\} \geq \sum_{m=1}^{\infty} \lambda_{m}\left\|\psi_{m}\right\|_{L^{2}(\Omega)}^{2},
$$

and also, $\|\Psi\|_{\mathcal{L}_{\underline{\lambda}}^{2}(\Omega)}<\infty$. We estimate

$$
\begin{aligned}
& \left\|T_{m} \psi_{k}\right\|_{L^{2}(\Omega)}^{2}=\left(\left(-\Delta+m^{2}\right) \psi_{k}, \psi_{k}\right)_{L^{2}(\Omega)} \\
& +m^{2}\left\|\psi_{k}\right\|_{L^{2}(\Omega)}^{2}-2 m\left(\sqrt{-\Delta+m^{2}} \psi_{k}, \psi_{k}\right)_{L^{2}(\Omega)} \leq \\
& \left\|\nabla \psi_{k}\right\|_{L^{2}(\Omega)}^{2}+2 m^{2}\left\|\psi_{k}\right\|_{L^{2}(\Omega)}^{2} \leq c(m)\left\|\psi_{k}\right\|_{H^{1}(\Omega)}^{2},
\end{aligned}
$$

where $c(m)$ is a mass dependent constant. Hence

$$
\left\|T_{m} \Psi\right\|_{\mathcal{L}_{\underline{\lambda}}^{2}(\Omega)}^{2}=\sum_{k=1}^{\infty} \lambda_{k}\left\|T_{m} \psi_{k}\right\|_{L^{2}(\Omega)}^{2} \leq c(m) \sum_{k=1}^{\infty} \lambda_{k}\left\|\psi_{k}\right\|_{H^{1}(\Omega)}^{2}<\infty .
$$

Lemma 4. The operator $T_{m}$ generates the group $e^{-i T_{m} t}, t \in \mathbb{R}$, of unitary operators on $\mathcal{L}_{\underline{\underline{\lambda}}}^{2}(\Omega)$.

Proof. For $\alpha, \beta \in \mathcal{L}_{\underline{\underline{\lambda}}}^{2}(\Omega)$ we compute the inner product

$$
\begin{aligned}
& \left(e^{-i T_{m} t} \alpha, e^{-i T_{m} t} \beta\right)_{\mathcal{L}_{\underline{\underline{\lambda}}}^{2}(\Omega)}=\sum_{k=1}^{\infty} \lambda_{k}\left(e^{-i T_{m} t} \alpha_{k}, e^{-i T_{m} t} \beta_{k}\right)_{L^{2}(\Omega)} \\
& =\sum_{k=1}^{\infty} \lambda_{k}\left(\alpha_{k}, \beta_{k}\right)_{L^{2}(\Omega)}=(\alpha, \beta)_{\mathcal{L}_{\mathbf{\lambda}}^{2}(\Omega)} .
\end{aligned}
$$

We rewrite the Schrödinger-Poisson system for $x \in \Omega$ into the form

$$
\begin{gathered}
\Psi_{t}=-i T_{m} \Psi+F[\Psi(x, t)], \text { where } F[\Psi]:=i^{-1} V[\Psi] \Psi, \\
-\Delta V[\Psi]=n[\Psi] \text {, where }\left.V\right|_{\partial \Omega}=0, \\
n[\Psi]=\sum_{k=1}^{\infty} \lambda_{k}\left|\psi_{k}\right|^{2}
\end{gathered}
$$

and prove the following auxiliary result.

Lemma 5. The map defined in (2.2) $F: \mathcal{H}_{\underline{\underline{\lambda}}}^{1}(\Omega) \rightarrow \mathcal{H}_{\underline{\underline{\lambda}}}^{1}(\Omega)$ is locally Lipschitz continuous. 

Then,

Proof. Let $\Psi, \Phi \in \mathcal{H}_{\underline{\underline{\lambda}}}^{1}(\Omega)$ with $\Psi=\left\{\psi_{k}\right\}_{k=1}^{\infty}, \Phi=\left\{\phi_{k}\right\}_{k=1}^{\infty}$ and $t \in[0, T]$.

$$
\begin{aligned}
& \|F[\Psi]-F[\Phi]\|_{\mathcal{H}_{\underline{\lambda}}^{1}(\Omega)}=\left\|i^{-1} V[\Psi] \Psi-i^{-1} V[\Phi] \Phi\right\|_{\mathcal{H}_{\underline{\lambda}}^{1}(\Omega)} \\
& =\|V[\Psi](\Psi-\Phi)+(V[\Psi]-V[\Phi]) \Phi\|_{\mathcal{H}_{\underline{\lambda}}^{1}(\Omega)} .
\end{aligned}
$$

This can be easily estimated above by means of Lemma 2 by

$$
C\|V[\Psi](\Psi-\Phi)\|_{\dot{\mathcal{H}}_{\underline{\underline{\lambda}}}^{1}(\Omega)}+C\|(V[\Psi]-V[\Phi]) \Phi\|_{\dot{\mathcal{H}}_{\underline{\underline{\lambda}}}^{1}(\Omega)},
$$

which equals

$$
C\left(\sum_{k=1}^{\infty} \lambda_{k}\left\|\nabla\left(V[\Psi]\left(\psi_{k}-\phi_{k}\right)\right)\right\|_{L^{2}(\Omega)}^{2}\right)^{\frac{1}{2}}+C\left(\sum_{k=1}^{\infty} \lambda_{k}\left\|\nabla\left((V[\Psi]-V[\Phi]) \phi_{k}\right)\right\|_{L^{2}(\Omega)}^{2}\right)^{\frac{1}{2}} .
$$

Here, $C$ denotes a finite, positive, universal constant. Clearly, we have $\left\|\nabla\left(V[\Psi]\left(\psi_{k}-\phi_{k}\right)\right)\right\|_{L^{2}(\Omega)}^{2} \leq 2\left\|(\nabla V[\Psi])\left(\psi_{k}-\phi_{k}\right)\right\|_{L^{2}(\Omega)}^{2}+2\left\|V[\Psi] \nabla\left(\psi_{k}-\phi_{k}\right)\right\|_{L^{2}(\Omega)}^{2}$.

By means of the Schwarz inequality this can be bounded above by

$$
C\|\nabla V[\Psi]\|_{L^{4}(\Omega)}^{2}\left\|\psi_{k}-\phi_{k}\right\|_{L^{6}(\Omega)}^{2}+2\|V[\Psi]\|_{L^{\infty}(\Omega)}^{2}\left\|\nabla\left(\psi_{k}-\phi_{k}\right)\right\|_{L^{2}(\Omega)}^{2} .
$$

By applying the Sobolev embedding theorems to these expressions, we arrive at

$$
C\|\Delta V[\Psi]\|_{L^{2}(\Omega)}^{2}\left\|\nabla\left(\psi_{k}-\phi_{k}\right)\right\|_{L^{2}(\Omega)}^{2} \leq C\|V[\Psi]\|_{H^{2}(\Omega)}^{2}\left\|\nabla\left(\psi_{k}-\phi_{k}\right)\right\|_{L^{2}(\Omega)}^{2} .
$$

To estimate the remaining term in (2.3), we use

$\left\|\nabla\left((V[\Psi]-V[\Phi]) \phi_{k}\right)\right\|_{L^{2}(\Omega)}^{2} \leq 2\left\|\nabla(V[\Psi]-V[\Phi]) \phi_{k}\right\|_{L^{2}(\Omega)}^{2}+2\left\|(V[\Psi]-V[\Phi]) \nabla \phi_{k}\right\|_{L^{2}(\Omega)}^{2}$.

The Schwarz inequality yields

$$
2\|\nabla(V[\Psi]-V[\Phi])\|_{L^{4}(\Omega)}^{2}\left\|\phi_{k}\right\|_{L^{4}(\Omega)}^{2}+2\|(V[\Psi]-V[\Phi])\|_{L^{\infty}(\Omega)}^{2}\left\|\nabla \phi_{k}\right\|_{L^{2}(\Omega)}^{2} .
$$

Applying the Sobolev embedding theorem along with the Hölder inequality to these expressions, we find

$$
C\|\Delta(V[\Psi]-V[\Phi])\|_{L^{2}(\Omega)}^{2}\left\|\phi_{k}\right\|_{L^{6}(\Omega)}^{2}+C\|\Delta(V[\Psi]-V[\Phi])\|_{L^{2}(\Omega)}^{2}\left\|\nabla \phi_{k}\right\|_{L^{2}(\Omega)}^{2} .
$$

From the Sobolev inequality used in the first of the two terms above we deduce the upper bound

$$
C\|V[\Psi]-V[\Phi]\|_{H^{2}(\Omega)}^{2}\left\|\nabla \phi_{k}\right\|_{L^{2}(\Omega)}^{2} .
$$

Therefore, for the norm of the difference $\|F[\psi]-F[\Phi]\|_{\mathcal{H}_{\underline{\lambda}}^{1}(\Omega)}$ we have the estimate from above as

$$
\begin{aligned}
& C\|V[\Psi]\|_{H^{2}(\Omega)}\left(\sum_{k=1}^{\infty} \lambda_{k}\left\|\nabla\left(\psi_{k}-\phi_{k}\right)\right\|_{L^{2}(\Omega)}^{2}\right)^{\frac{1}{2}} \\
& +C\|V[\Psi]-V[\Phi]\|_{H^{2}(\Omega)}\left(\sum_{k=1}^{\infty} \lambda_{k}\left\|\nabla \phi_{k}\right\|_{L^{2}(\Omega)}^{2}\right)^{\frac{1}{2}},
\end{aligned}
$$

which obviously equals to

$$
C\|V[\Psi]\|_{H^{2}(\Omega)}\|\Psi-\Phi\|_{\dot{\mathcal{H}}_{\underline{\underline{\lambda}}}^{1}(\Omega)}+C\|V[\Psi]-V[\Phi]\|_{H^{2}(\Omega)}\|\Phi\|_{\dot{\mathcal{H}}_{\underline{\underline{\lambda}}}^{1}(\Omega)} .
$$

Let us apply the Poincaré and the Schwarz inequalities to estimate the Sobolev norm of the potential function as

$$
\|V[\Psi]\|_{H^{2}(\Omega)} \leq C\|\Delta V\|_{L^{2}(\Omega)}=C\|n[\Psi]\|_{L^{2}(\Omega)} .
$$


Hence, our goal is to estimate the appropriate norm of the particle concentration. From the Schwarz inequality,

$$
\|n[\Psi]\|_{L^{2}(\Omega)}^{2}=\sum_{k, l=1}^{\infty} \lambda_{k} \lambda_{l}\left(\left|\psi_{k}\right|^{2},\left|\psi_{l}\right|^{2}\right)_{L^{2}(\Omega)} \leq\left(\sum_{k=1}^{\infty} \lambda_{k}\left\|\psi_{k}\right\|_{L^{4}(\Omega)}^{2}\right)^{2} .
$$

and using the Hölder inequality along with the Sobolev inequality,

$$
\|n[\Psi]\|_{L^{2}(\Omega)} \leq C \sum_{k=1}^{\infty} \lambda_{k}\left\|\psi_{k}\right\|_{L^{6}(\Omega)}^{2} \leq C \sum_{k=1}^{\infty} \lambda_{k}\left\|\nabla \psi_{k}\right\|_{L^{2}(\Omega)}^{2} .
$$

Hence, we arrive at the estimates for the particle concentration and the norms on the potential function,

$$
\|n[\Psi]\|_{L^{2}(\Omega)} \leq C\|\Psi\|_{\mathcal{H}_{\underline{\underline{\lambda}}}^{1}(\Omega)}^{2}, \quad\|V[\Psi]\|_{H^{2}(\Omega)} \leq C\|\Psi\|_{\mathcal{H}_{\underline{\underline{\lambda}}}^{1}(\Omega)}^{2}
$$

with $\|\cdot\|_{\mathcal{H}_{\underline{\lambda}}^{1}(\Omega)}$ and $\|\cdot\|_{\mathcal{H}_{\underline{\lambda}}^{1}(\Omega)}$ equivalent via Lemma 2. Evidently,

$$
W:=V[\Psi]-V[\Phi]
$$

satisfies the Poisson equation,

$$
-\Delta W=n[\Psi]-n[\Phi],\left.\quad W\right|_{\partial \Omega}=0,
$$

and Dirichlet boundary conditions. Applying the Poincaré inequality along with the Schwarz inequality, we arrive at

$$
\|W\|_{H^{2}(\Omega)}^{2} \leq C\|\Delta W\|_{L^{2}(\Omega)}^{2},
$$

such that

$$
\|W\|_{H^{2}(\Omega)} \leq C\|n[\Psi]-n[\Phi]\|_{L^{2}(\Omega)} .
$$

We will use the trivial inequality

$$
|n[\Psi]-n[\Phi]| \leq \sum_{k=1}^{\infty} \lambda_{k}\left(\left|\psi_{k}\right|+\left|\phi_{k}\right|\right)\left|\psi_{k}-\phi_{k}\right| .
$$

The Schwarz inequality applied twice yields

$$
\begin{aligned}
& \|n[\Psi]-n[\Phi]\|_{L^{2}(\Omega)}^{2} \leq\left(\sum_{k=1}^{\infty} \lambda_{k} \sqrt{\int_{\Omega}\left(\left|\psi_{k}\right|+\left|\phi_{k}\right|\right)^{2}\left|\psi_{k}-\phi_{k}\right|^{2} d x}\right)^{2} \\
& \leq\left(\sum_{k=1}^{\infty} \lambda_{k}\left\|\left|\psi_{k}\right|+\left|\phi_{k}\right|\right\|_{L^{4}(\Omega)}\left\|\psi_{k}-\phi_{k}\right\|_{L^{4}(\Omega)}\right)^{2} \\
& \leq\left(\sum_{k=1}^{\infty} \lambda_{k}\left(\left\|\psi_{k}\right\|_{L^{4}(\Omega)}+\left\|\phi_{k} \mid\right\|_{L^{4}(\Omega)}\right)\left\|\psi_{k}-\phi_{k}\right\|_{L^{4}(\Omega)}\right)^{2},
\end{aligned}
$$

and using it again gives

$$
\sum_{k=1}^{\infty} \lambda_{k}\left(\left\|\psi_{k}\right\|_{L^{4}(\Omega)}+\left\|\phi_{k} \mid\right\|_{L^{4}(\Omega)}\right)^{2} \sum_{s=1}^{\infty} \lambda_{s}\left\|\psi_{s}-\phi_{s}\right\|_{L^{4}(\Omega)}^{2} .
$$

Applying the Hölder and Sobolev inequalities, we arrive at

$$
C \sum_{k=1}^{\infty} \lambda_{k}\left(\left\|\nabla \psi_{k}\right\|_{L^{2}(\Omega)}^{2}+\left\|\nabla \phi_{k}\right\|_{L^{2}(\Omega)}^{2}\right) \sum_{s=1}^{\infty} \lambda_{s}\left\|\nabla \psi_{s}-\nabla \phi_{s}\right\|_{L^{2}(\Omega)}^{2} .
$$


This quantity can be easily estimated above by

$$
C\left(\sum_{k=1}^{\infty} \lambda_{k}\left\|\psi_{k}\right\|_{H^{1}(\Omega)}^{2}+\sum_{l=1}^{\infty} \lambda_{l}\left\|\phi_{l}\right\|_{H^{1}(\Omega)}^{2}\right) \sum_{s=1}^{\infty} \lambda_{s}\left\|\psi_{s}-\phi_{s}\right\|_{H^{1}(\Omega)}^{2},
$$

which clearly equals to

$$
C\left(\|\Psi\|_{\mathcal{H}_{\underline{\underline{\lambda}}}^{1}(\Omega)}^{2}+\|\Phi\|_{\mathcal{H}_{\underline{\underline{\lambda}}}^{1}(\Omega)}^{2}\right)\|\Psi-\Phi\|_{\mathcal{H}_{\underline{\underline{\lambda}}}^{1}(\Omega)}^{2} .
$$

Therefore,

$$
\|n[\Psi]-n[\Phi]\|_{L^{2}(\Omega)} \leq C\left(\|\Psi\|_{\mathcal{H}_{\underline{\underline{\lambda}}}^{1}(\Omega)}+\|\Phi\|_{\mathcal{H}_{\underline{\underline{\lambda}}}^{1}(\Omega)}\right)\|\Psi-\Phi\|_{\mathcal{H}_{\underline{\underline{\lambda}}}^{1}(\Omega)}
$$

and

$$
\|V[\Psi]-V[\Phi]\|_{H^{2}(\Omega)} \leq C\left(\|\Psi\|_{\mathcal{H}_{\underline{\underline{\lambda}}}^{1}(\Omega)}+\|\Phi\|_{\mathcal{H}_{\underline{\underline{\lambda}}}^{1}(\Omega)}\right)\|\Psi-\Phi\|_{\mathcal{H}_{\underline{\underline{\lambda}}}^{1}(\Omega)} .
$$

Collecting the estimates above, we arrive at

$$
\|F[\Psi]-F[\Phi]\|_{\mathcal{H}_{\underline{\underline{\lambda}}}^{1}(\Omega)} \leq C\left(\|\Psi\|_{\mathcal{H}_{\underline{\underline{\lambda}}}^{1}(\Omega)}^{2}+\|\Phi\|_{\mathcal{H}_{\underline{\underline{\lambda}}}^{1}(\Omega)}^{2}\right)\|\Psi-\Phi\|_{\mathcal{H}_{\underline{\underline{\lambda}}}^{1}(\Omega)}
$$

which completes the proof of the lemma.

From standard arguments (see for instance Theorem 1.7 of [7]) thus follows that the above Schrödinger-Poisson system admits a unique mild solution $\Psi$ in $\mathcal{H}_{\underline{\lambda}}^{1}(\Omega)$ on a time interval $[0, T)$, for some $T>0$, satisfying the integral equation

$$
\Psi(t)=e^{-i T_{m} t} \Psi(0)+e^{-i T_{m} t} \int_{0}^{t} e^{i T_{m} s} F[\Psi(s)] d s
$$

in $\mathcal{H}_{\underline{\underline{\lambda}}}^{1}(\Omega)$. Moreover,

$$
\lim _{t \nearrow T}\|\Psi(t)\|_{\mathcal{H}_{\underline{\underline{\lambda}}}^{1}(\Omega)}=\infty
$$

if $T$ is finite. We also note that $\Psi$ is a unique strong solution in $\mathcal{L}_{\underline{\lambda}}^{2}(\Omega)$. We shall next prove that this solution is in fact global in time. First we prove the following lemma.

LEMMA 6. Suppose for the unique mild solution (2.4) of the SchrödingerPoisson system (1.6)-(1.8) that $\left\{\psi_{k}(x, 0)\right\}_{k=1}^{\infty}$ at $t=0$ forms a complete orthonormal system in $L^{2}(\Omega)$. Then, for any $t \in[0, T)$, the set $\left\{\psi_{k}(x, t)\right\}_{k=1}^{\infty}$ remains a complete orthonormal system in $L^{2}(\Omega)$. Moreover, the $\mathcal{L}_{\underline{\underline{\lambda}}}^{2}(\Omega)$-norm is preserved, $\|\Psi(x, t)\|_{\mathcal{L}_{\underline{\underline{\lambda}}}^{2}(\Omega)}=\|\Psi(x, 0)\|_{\mathcal{L}_{\underline{\underline{\lambda}}}^{2}(\Omega)}, t \in[0, T)$.

Proof. Given the solution $\Psi(t)$ of the Schrödinger-Poisson system on $[0, T)$, we obtain the time-dependent one-particle Hamiltonian

$$
H_{V_{\Psi}}(t)=T_{m}+V_{\Psi}(t, x)
$$

where the potential $V_{\Psi}$ solves $-\Delta V_{\Psi}(t, x)=n[\Psi(t)]$ with Dirichlet boundary conditions, see (1.2). Accordingly, the components of $\Psi(t)$ solve the linear, nonautonomous Schrödinger equation $i \partial_{t} \psi_{k}(t, x)=H_{V_{\Psi}}(t) \psi_{k}(t, x)$, for $k \in \mathbb{N}$, on the time interval $[0, T)$. We thus have, for $t \in[0, T)$,

$$
\psi_{k}(x, t)=\left(e^{-i \int_{0}^{t} H_{V_{\Psi}}(\tau) d \tau} \psi_{k}\right)(x, 0), k \in \mathbb{N},
$$

and therefore

$$
\left(\psi_{k}(x, t), \psi_{l}(x, t)\right)_{L^{2}(\Omega)}=\left(e^{-i \int_{0}^{t} H_{V_{\Psi}}(\tau) d \tau} \psi_{k}(x, 0), e^{-i \int_{0}^{t} H_{V_{\Psi}}(\tau) d \tau} \psi_{l}(x, 0)\right)_{L^{2}(\Omega)}=
$$




$$
=\left(\psi_{k}(x, 0), \psi_{l}(x, 0)\right)_{L^{2}(\Omega)}=\delta_{k, l}, \quad k, l \in \mathbb{N},
$$

where $\delta_{k, l}$ stands for the Kronecker symbol. Obviously, for $k \in \mathbb{N}$,

$$
\left\|\psi_{k}(x, t)\right\|_{L^{2}(\Omega)}^{2}=\left\|\psi_{k}(x, 0)\right\|_{L^{2}(\Omega)}^{2},
$$

such that for $t \in[0, T)$, the $\mathcal{L}_{\underline{\underline{\lambda}}}^{2}(\Omega)$-norm is conserved,

$$
\begin{aligned}
& \|\Psi(x, t)\|_{\mathcal{L}_{\underline{\lambda}}^{2}(\Omega)}=\left(\sum_{k=1}^{\infty} \lambda_{k}\left\|\psi_{k}(x, t)\right\|_{L^{2}(\Omega)}^{2}\right)^{\frac{1}{2}} \\
& =\left(\sum_{k=1}^{\infty} \lambda_{k}\left\|\psi_{k}(x, 0)\right\|_{L^{2}(\Omega)}^{2}\right)^{\frac{1}{2}}=\|\Psi(x, 0)\|_{\mathcal{L}_{\underline{\lambda}}^{2}(\Omega)} .
\end{aligned}
$$

Let us consider an arbitrary function $f(x) \in L^{2}(\Omega)$. Clearly, we have the expansion

$$
f(x)=\sum_{k=1}^{\infty}\left(f(y), \psi_{k}(y, 0)\right)_{L^{2}(\Omega)} \psi_{k}(x, 0)
$$

and similarly

$$
e^{i \int_{0}^{t} H_{V_{\Psi}}(\tau) d \tau} f(x)=\sum_{k=1}^{\infty}\left(e^{i \int_{0}^{t} H_{V_{\Psi}}(\tau) d \tau} f(y), \psi_{k}(y, 0)\right)_{L^{2}(\Omega)} \psi_{k}(x, 0) .
$$

Thus, by means of (2.5) we arrive at the expansion

$$
f(x)=\sum_{k=1}^{\infty}\left(f(y), \psi_{k}(y, t)\right)_{L^{2}(\Omega)} \psi_{k}(x, t)
$$

for $t \in[0, T)$.

Furthermore, we have conservation of energy for solutions to the SchrödingerPoisson system in the following sense.

Lemma 7. For the unique mild solution (2.4) of the Schrödinger-Poisson system (1.6)-(1.8) and for any value of time $t \in[0, T)$ we have the identity

$\|\Psi(x, t)\|_{\mathcal{H}_{\underline{\lambda}}^{1 / 2}(\Omega)}^{2}+\frac{1}{2}\|\nabla V[\Psi(x, t)]\|_{L^{2}(\Omega)}^{2}=\|\Psi(x, 0)\|_{\mathcal{H}_{\underline{\lambda}}^{1 / 2}(\Omega)}^{2}+\frac{1}{2}\|\nabla V[\Psi(x, 0)]\|_{L^{2}(\Omega)}^{2}$.

Proof. Complex conjugation of the Schrödinger-Poisson system (1.6) yields

$$
-i \frac{\partial \bar{\psi}_{k}}{\partial t}=T_{m} \bar{\psi}_{k}+V[\psi] \bar{\psi}_{k}, \quad k \in \mathbb{N} .
$$

Adding the $k$-th equation of the original system (1.6) multiplied by $\frac{\partial \bar{\psi}_{k}}{\partial t}$, and the $k$-th equation in (2.6) multiplied by $\frac{\partial \psi_{k}}{\partial t}$, we obtain

$$
\frac{\partial}{\partial t}\left\|T_{m}^{\frac{1}{2}} \psi_{k}\right\|_{L^{2}(\Omega)}^{2}+\int_{\Omega} V[\psi] \frac{\partial}{\partial t}\left|\psi_{k}\right|^{2} d x=0, \quad k \in \mathbb{N} .
$$

Thus, multiplying by $\lambda_{k}$, and summing over $k$, we find

$$
\frac{\partial}{\partial t}\|\Psi(x, t)\|_{\dot{\mathcal{H}}_{\underline{\lambda}}^{1 / 2}(\Omega)}^{2}+\int_{\Omega} V[\Psi(x, t)] \frac{\partial}{\partial t} n[\Psi(x, t)] d x=0 .
$$


One can easily verify the identity

$$
\frac{\partial}{\partial t}\|\nabla V[\Psi(x, t)]\|_{L^{2}(\Omega)}^{2}=2 \int_{\Omega} V[\Psi(x, t)] \frac{\partial}{\partial t} n[\Psi(x, t)] d x
$$

which we substitute in (2.7) to complete the proof of the lemma.

With the auxiliary statements proven above at our disposal, we may now prove our main result, Theorem 1 .

Proof of TheOrem 1. The proof follows from the blow-up alternative and conservation laws. It follows from Lemma 7 that $\|\Psi(t)\|_{\dot{\mathcal{H}}_{\underline{\lambda}}^{1 / 2}(\Omega)}$ is bounded from above uniformly in time,

$$
\begin{aligned}
& \|\Psi(t)\|_{\dot{\mathcal{H}}_{\underline{\lambda}}^{1 / 2}(\Omega)}^{2} \leq\|\Psi(t)\|_{\dot{\mathcal{H}}_{\underline{\lambda}}^{1 / 2}(\Omega)}^{2}+\frac{1}{2}\|\nabla V[\Psi(t)]\|_{L^{2}(\Omega)}^{2} \\
& =\|\Psi(0)\|_{\dot{\mathcal{H}}_{\underline{\underline{\lambda}}}^{1 / 2}(\Omega)}^{2}+\frac{1}{2}\|\nabla V[\Psi(0)]\|_{L^{2}(\Omega)}^{2} .
\end{aligned}
$$

We need to bound $\|\Psi(t)\|_{\dot{\mathcal{H}}_{\underline{\lambda}}^{1}(\Omega)}$. We recall the mild solution of the SchrödingerPoisson system (1.6)-(1.8), given by

$$
\Psi(t)=e^{-i T_{m} t} \Psi(0)+e^{-i T_{m} t} \int_{0}^{t} e^{i T_{m} s} F[\Psi(s)] d s,
$$

which implies

$$
\|\Psi(t)\|_{\mathcal{H}_{\underline{\lambda}}^{1}(\Omega)} \leq\|\Psi(0)\|_{\mathcal{H}_{\underline{\underline{\lambda}}}^{1}(\Omega)}+\int_{0}^{t}\|F[\Psi(s)]\|_{\mathcal{H}_{\underline{\underline{\lambda}}}^{1}(\Omega)} .
$$

From Lemma 2, we have

$$
\begin{aligned}
\|F[\Psi]\|_{\mathcal{H}_{\underline{\lambda}}^{1}(\Omega)} & =\|V[\Psi] \Psi\|_{\mathcal{H}_{\underline{\lambda}}^{1}(\Omega)} \leq C\|V[\Psi] \Psi\|_{\dot{\mathcal{H}}_{\underline{\lambda}}^{1}(\Omega)} \\
& \leq C\left(\sum_{k=1}^{\infty} \lambda_{k}\left\|\nabla\left(V[\Psi] \psi_{k}\right)\right\|_{L^{2}(\Omega)}^{2}\right)^{1 / 2} .
\end{aligned}
$$

Now,

$$
\begin{aligned}
\|\nabla(V[\psi] \psi)\|_{L^{2}(\Omega)}^{2} & \leq\left\|\nabla V[\Psi] \psi_{k}\right\|_{L^{2}(\Omega)}^{2}+\left\|V[\Psi] \nabla \psi_{k}\right\|_{L^{2}(\Omega)}^{2} \\
& \leq\|\nabla V[\Psi]\|_{L^{6}(\Omega)}^{2}\left\|\psi_{k}\right\|_{L^{3}(\Omega)}^{2}+\|V[\Psi]\|_{L^{\infty}(\Omega)}^{2}\left\|\nabla \psi_{k}\right\|_{L^{2}(\Omega)}^{2} \\
& \leq\|\nabla V[\Psi]\|_{L^{6}(\Omega)}^{2}\left\|\psi_{k}\right\|_{H^{1 / 2}(\Omega)}^{2}+\|V[\Psi]\|_{L^{\infty}(\Omega)}^{2}\left\|\psi_{k}\right\|_{H^{1}(\Omega)}^{2}
\end{aligned}
$$

where we have used Hölder's inequality in the second line and the Sobolev inequality

$$
\|f\|_{L^{\frac{6}{3-2 p}}(\Omega)} \leq C\|f\|_{H^{p}(\Omega)}
$$


in the last line. To evaluate $\|\nabla V[\Psi]\|_{L^{6}(\Omega)}$, recall that $\Delta V[\Psi]=-n[\Psi]$. Applying Hölder's and Sobolev inequalities, we get

$$
\begin{aligned}
\|\nabla V[\Psi]\|_{L^{6}(\Omega)}^{2} & \leq C\|\nabla V[\Psi]\|_{H^{1}(\Omega)}^{2} \leq C\|n[\Psi]\|_{L^{2}(\Omega)}^{2} \\
& \leq C \sum_{k, l=1}^{\infty} \lambda_{k} \lambda_{l}\left(\left|\psi_{k}\right|^{2},\left|\psi_{l}\right|^{2}\right)_{L^{2}(\Omega)} \leq C \sum_{k, l=1}^{\infty} \lambda_{k} \lambda_{l}\left\|\psi_{k} \psi_{l}\right\|_{L^{2}(\Omega)}^{2} \\
& \leq C \sum_{k, l=1}^{\infty} \lambda_{k} \lambda_{l}\left\|\psi_{k}\right\|_{L^{6}(\Omega)}^{2}\left\|\psi_{l}\right\|_{L^{3}(\Omega)}^{2} \\
& \leq C\left(\sum_{k=1}^{\infty} \lambda_{k}\left\|\psi_{k}\right\|_{H^{1}(\Omega)}^{2}\right)\left(\sum_{l=1}^{\infty} \lambda_{l}\left\|\psi_{l}\right\|_{H^{1 / 2}(\Omega)}^{2}\right) \\
& \leq C\|\Psi\|_{\mathcal{H}_{\underline{\lambda}}^{1}(\Omega)}^{2}\|\Psi\|_{\dot{\mathcal{H}}_{\underline{\lambda}}^{1 / 2}(\Omega)}^{2}
\end{aligned}
$$

We now estimate $\|V[\Psi]\|_{L^{\infty}(\Omega)}$. The Sobolev inequality implies

$$
\|V[\Psi]\|_{L^{\infty}(\Omega)}^{2} \leq C\left\|\left.|| p\right|^{-1 / 2} n[\Psi]\right\|_{L^{2}(\Omega)}^{2} .
$$

We claim that $\left\||p|^{-1 / 2} n[\Psi]\right\|_{L^{2}(\Omega)}$ is controlled by $\|\Psi\|_{\dot{\mathcal{H}}_{\underline{\lambda}}^{1 / 2}(\Omega)}$.

$$
\begin{aligned}
\left\||p|^{-1 / 2} n[\Psi]\right\|_{L^{2}(\Omega)}^{2} & =\left(n[\Psi],|p|^{-1} n[\Psi]\right)_{L^{2}(\Omega)} \leq\|n[\Psi]\|_{L^{3 / 2}(\Omega)}\left\|\left.p\right|^{-1} n[\Psi]\right\|_{L^{3}(\Omega)} \\
& \leq C\|\Psi\|_{L^{3}(\Omega)}^{2}\left\||p|^{-1} n[\Psi]\right\|_{H^{1 / 2}(\Omega)} \\
& \leq C\|\Psi\|_{H^{1 / 2}(\Omega)}^{2}\left\||p|^{-1 / 2} n[\Psi]\right\|_{L^{2}(\Omega)},
\end{aligned}
$$

where we have used Hölder's inequality in the first line, and the Sobolev inequality in the second line. It follows that

$$
\left\||p|^{-1 / 2} n[\Psi]\right\|_{L^{2}(\Omega)} \leq C\|\Psi\|_{\dot{\mathcal{H}}_{\underline{\lambda}}^{1 / 2}(\Omega)}^{2},
$$

and hence

$$
\|V[\Psi]\|_{L^{\infty}(\Omega)}^{2} \leq C\|\Psi\|_{\dot{\mathcal{H}}_{\underline{\lambda}}^{1 / 2}(\Omega)}^{4}
$$

Combining the above estimates yields

$$
\|F[\Psi]\|_{\dot{\mathcal{H}}_{\underline{\underline{\lambda}}}^{1}(\Omega)} \leq C\|\Psi\|_{\dot{\mathcal{H}}_{\underline{\underline{\lambda}}}^{1 / 2}(\Omega)}^{2}\|\Psi\|_{\dot{\mathcal{H}}_{\underline{\underline{x}}}^{1}(\Omega)}
$$

This implies

$$
\|\Psi(t)\|_{\mathcal{H}_{\underline{\lambda}}^{1}(\Omega)} \leq\|\Psi(0)\|_{\mathcal{H}_{\underline{\underline{\lambda}}}^{1}(\Omega)}+\int_{0}^{t} C_{0}\|\Psi(s)\|_{\mathcal{H}_{\underline{\lambda}}^{1}(\Omega)},
$$

where $C_{0}$ is a constant proportional to the initial energy

$$
\|\Psi(0)\|_{\dot{\mathcal{H}}_{\underline{\lambda}}^{1 / 2}(\Omega)}^{2}+\frac{1}{2}\|\nabla V[\Psi(0)]\|_{L^{2}(\Omega)}^{2} .
$$

By Gronwall's lemma,

$$
\|\Psi(t)\|_{\mathcal{H}_{\underline{\underline{\lambda}}}^{1}(\Omega)} \leq C_{1} e^{C_{2} t}, \quad t>0
$$

By the blow-up alternative, this implies that the Schrödinger-Poisson system is globally well-posed in $\mathcal{H}_{\underline{\underline{\lambda}}}^{1}(\Omega)$. 


\section{Acknowledgements}

V.V. thanks J. Colliander, R. Jerrard, and I.M. Sigal for stimulating discussions. In particular, the idea to pursue this problem originates from discussions and seminars on related topics initiated by I.M. Sigal that V.V. was part of, around 2007 at the University of Toronto. The work of T.C. was supported by NSF grant DMS1009448. W.A.S. acknowledges the support of NSERC discovery grant and USASK start-up fund.

\section{References}

[1] G.L. Aki, P.A. Markowich, C. Sparber. Classical limit for semirelativistic Hartree systems. J.Math.Phys. 49 (2008), no. 10, 102110, 10pp.

[2] I. Anapolitanos. Rate of convergence towards the Hartree-von Neumann limit in the meanfield regime. Lett. Math. Phys. 98 (2010), 1-31.

[3] I. Anapolitanos, I. M. Sigal. The Hartree-von Neumann limit of many body dynamics. Preprint [http://arxiv.org/abs/0904.4514]

[4] F. Brezzi, P. Markowich. The three-dimensional Wigner-Poisson problem: existence, uniqueness and approximation. Math. Methods Appl. Sci. 14 (1991), no. 1, 35-61.

[5] E. Lenzmann. Well-posedness for semi-relativistic Hartree equations of critical type. Math. Phys. Anal. Geom. 10 (2007), no.1, 43-64.

[6] P.A. Markowich, G. Rein, G. Wolansky. Existence and nonlinear stability of stationary states of the Schrödinger- Poisson system. J.Statist. Phys. 106 (2002), no. 5-6, 1221-1239.

[7] A. Pazy. Semigroups of Linear Operators and Applications to Partial Differential Equations, Springer, Berlin (1983).

[8] H. Steinrück. The one-dimensional Wigner-Poisson problem and its relation to the Schrödinger- Poisson problem. SIAM J. Math. Anal. 22 (1991), no. 4, 957-972.

Department of Mathematics and Statistics, University of Saskatchewan, SaskaTOON, SK, S7N 5E6, CANADA

E-mail address: walid.abousalem@usask.ca

Department of Mathematics, University of Texas at Austin, Austin, TX, 78712, USA

E-mail address: tc@math.utexas.edu

University of Cape Town, Department of Mathematics and Applied Mathematics, Private Bag, Rondebosch 7701, South Africa

E-mail address: Vitali.Vougalter@uct.ac.za 